Group A due to more severe disease and higher rate of ulcerative colitis, table 1 shows the diagnostic breakdowns from both groups following endoscopy. The IBD pick up was significantly higher in Group A with $70 \%$ vs 42\%. Endoscopy DNA rate was twice as high in Group B $(n=6)$. The direct to IBD endoscopy pathway resulted in 50 less initial IBD consultant clinics (100\% reduction) with a follow-up shift from IBD consultant to IBD nurse clinics.

Conclusion Triaging patients referred with suspected IBD directly to IBD physician delivered endoscopy resulted in more than a 26-week reduction in time to diagnosis and treatment while saving $100 \%$ of initial IBD consultant clinics. IBD pick up was high at $70 \%$ in direct to IBD endoscopy group, identifying a higher-need IBD population. Triaging GP referrals with suspected IBD direct to IBD endoscopy delivers rapid assessment and treatment.

\section{O70 PATIENT REPORTED EXPERIENCES THROUGH PBC FOUNDATION APP: WHAT IMPACTS UPON A PBC PATIENT'S EXPERIENCE?}

${ }^{1}$ Robert Mitchell-Thain*, ${ }^{2}$ Andrew Yeoman, ${ }^{3}$ Vinod Hegade. ${ }^{1} P B C$ Foundation, Edinburgh, UK; ${ }^{2}$ Hepatologist, Gwent Liver Unit, Newport, UK; ${ }^{3}$ Consultant Hepatologist, Leeds Liver Unit, Leeds, UK

\subsection{6/gutjnl-2020-bsgcampus.70}

Intro The $\mathrm{PBC}$ Foundation is a UK-based patient support organisation which supports patients with $\mathrm{PBC}$ in over 75 countries, affected by Primary Biliary Cholangitis (PBC). The PBC Foundation has developed a self-management App to conduct surveys to anonymously record Patient Recorded Outcome Measurements (PROMS) and Patient Recorded Experience Measures (PREMS). This App went live in 2019 with patients from 20 different countries registered and over 3200 downloads.

The aim of this study is to report the early aggregate findings of the first surveys conducted through the App and provide future directions on how this may be utilised in improving PBC patient care.

Methods In Nov 2019, we set two App-based surveys. The surveys have $n=189$ and $n=171$, respectively.

Results We found, firstly, that age has an impact upon the quality of care experienced by PBC patients. We asked patients, 'If, for any reason, you have not fully responded to Urso, have you been offered an additional second-line treatment?' The answer varied between age groups:

31-40 yrs, 31\% no: $41-50$ yrs, 47\% no: 51-60 yrs, 54\% no: $61-70$ yrs, $48 \%$ no: $70+y r s, 83 \%$ no, with an overall rate of $49.5 \%$ no.

We also asked, 'If, for any reason, you have not fully responded to Urso, has your clinician discussed second-line treatments with you?' Again, the answers were low at baseline, and correlated with patient age:

31-40 yrs, 27\% no: $41-50$ yrs, $38 \%$ no: $51-60$ yrs, $46 \%$ no: $61-70$ yrs, $45 \%$ no: $70+y r s, 50 \%$ no, with an overall rate of $41.5 \%$ no.

In terms of symptom management, one factor that appears to impact upon the patient experience is whether they see a gastroenterologist or a hepatologist. We asked, 'Has your clinician offered any advice or treatments to manage your fatigue. Interestingly, only $12.7 \%$ of patients experienced no fatigue. Of the $87.3 \%$ who did experience fatigue, only $17 \%$ of
Gastro patients and 23\% of Hep patients answered, 'yes.' When asked, 'Has your clinician offered any advice or treatment to manage your itch?' $67 \%$ of Gastro patients and $47 \%$ of Hep patients said yes.

Conclusions The data strongly suggests that there is significant unmet need, in terms of both disease management and symptom management, in PBC patients: particularly in those patients most in need of clinical intervention. The PBC Foundation App surveys provide useful insights into issues prevalent in the care of patients.

\section{INTRODUCING AN ELECTRONIC ALCOHOL SCREENING TOOL IMPROVES ALCOHOL MISUSE PATIENT IDENTIFICATION REDUCES LENGTH OF STAY}

Julius Mukarati*. Uhow Nhs Trust, Coventry, UK

\subsection{6/gutjnl-2020-bsgcampus.71}

Introduction The Modified Single Alcohol Screening Questionnaire was a paper-based tool recorded in the Emergency Department booklet which was being under-utilised and difficult to audit. We had suspected that the length of stay was high in this patient group due to lack of identification resulting in missed opportunities to intervene effectively. Therefore we conducted an audit considering a consecutive random 100 patients retrospectively in 2017 based on the referral received showing that only $40-60 \%$ were being referred into the Alcohol Liaison Service thus we sought to improve the identification through the introduction of an electronic alcohol screening tool.

Method The electronic alcohol tool has an inbuilt alcohol assessment module on Vital Pac taking the nurse or clinician through a series of relevant alcohol questions in relation to the admission and derives at a score which determines the risk levels regarding that patient. The tool was implemented initially on a trial basis and fully implemented following staff training. The tool was then re-audited in 2019 reviewing electronic records stored on Vital Pac and our Clinical Results Reporting System (CRRS).

Results The results post implementation show an increase in compliancy which rose to $70-90 \%$ of patient being screened for alcohol. The number of referrals and patient contact also increased by approximately $7 \%$ as the patient contact rose from $47 \%$ in 2016 to $54 \%$ in 2019, which, meant intervention was more timely therefore minimising risk. The total referrals in 2019 were 823 patients in comparison to 760 patients in 2018 showing a $4 \%$ increase in referrals due to the electronic tool prompting staff to refer patients identified as being at risk. The tool improved efficiency with robust data collection for audit. Bed days reduced per month from an average of $6-3$ bed days.

Conclusion The alcohol electronic screening reduced omissions and increased contact, minimising risk to patients. Cost effectiveness was noted through reduction in bed days due to effective patient management. The tool aided in promoting quality and safety in patient care delivery through, managing this patient group in a timely manner, improvement quality and suitable intervention, reducing risk and harm to this patient group, empowering clinical staff to recognise this patient group and be confident to manage their care effectively through knowledge and awareness as well as improve pathways for this patient group and trust policies. 